\title{
Evolution of Techniques for Question Answering over Knowledge Base: A Survey
}

\author{
Ashish Salunkhe \\ Pimpri Chinchwad College of Engineering and Research \\ Pune, India
}

\begin{abstract}
In this paper, a brief study of the advancements in the Question Answering domain as a type of information retrieval system is presented. Question Answering systems are responsible to provide answers to the questions proposed over a knowledge base in natural language to retrieve the required information. The promising results achieved in Question Answering in Natural Language Processing are discussed. The aim is to cover a concise yet complete understanding of the advances in Question Answering Systems classified based on domain and question type and brief information about metrics used to evaluate the system.
\end{abstract}

\section{Keywords}

Data mining, text mining, question answering, classification, named entity recognition, neural networks, pos tagging

\section{INTRODUCTION}

Information pertaining to numerous domains containing numerous facts is growing at a rapid rate. While this information is easily extracted and analyzed by the experts, the non-experts can't take the benefit of the same. [1], [2]. This in turn has set the platform of developing and modeling question answering and keyword search tools for Information Retrieval [3]-[6]. Question Answering Systems incorporate natural language statements or questions to retrieve information from complete document where specific pieces of information are returned as an answer. Using some ranking scheme, QA systems combine data retrieval with knowledge extraction methods to classify a range of likely candidates and then generate final answers. [7]. Question Answering has always been a promising-research area in Natural Language Processing. Question Answering systems have been widely used in various applications - information retrieval, named entity recognition, dialog systems [8]. In recent years, there has been significant increase in the amount of data being generated on a daily basis. Users bear questions for which they expect an exact, precise and short response as an answer. The response is expected to be given in the natural language without being limited to a particular query language, query training rules or even a specific information domain.

\section{GENERAL ARCHITECTURE}

A typical question answering system consists of following phases: Question Classification, Information Retrieval and Analysis and Answer Analysis. Question Classification is the initial phase where questions are classified by keyword extraction and semantic analysis. Queries are fired to retrieve information along with information extraction algorithms. Ranking of passages and information retrieval recall methods are used to boost information retrieval and analysis process. Answer extraction and analysis is the last phase in question answering system, which is the discrimination mark [25].

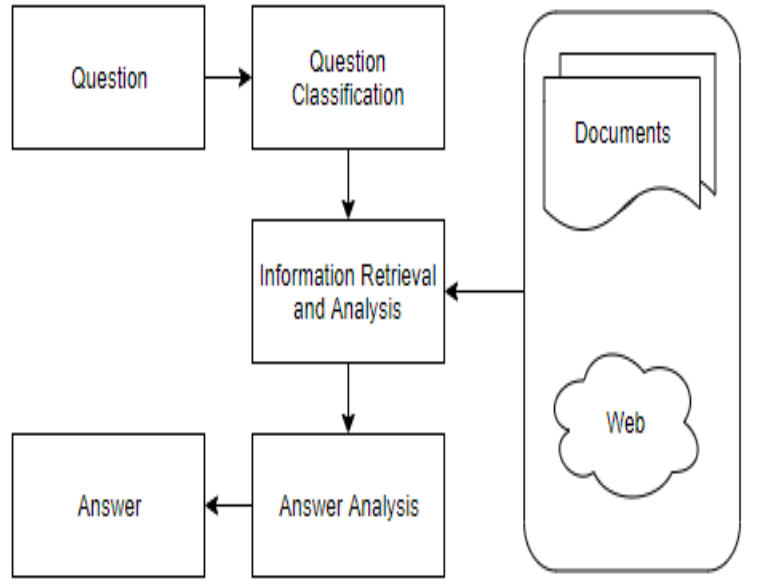

Fig. 1: General Architecture

\section{TYPES OF QUESTION ANSWERING SYSTEMS}

\subsection{Based on Domain}

Open domain Question Answering system: Systems providing short answers in natural language and no restricted to a particular domain are Open Domain Question Answering Systems. The Open Domain QA depends on information retrieved from World Wide Web and universal ontology and these sources are responsible for answers to every question asked. These systems require general vocabulary avoiding domain specific vocabulary. Open-domain data set such as Wikipedia is used for question answering. There has been significant amount of work done in Open Domain Question Answering. The data sets include Quasar [26], MS Marco [27], Triviaqa [28], Searchqa [29], SQuAD [30], Hotpotqa [31]. Research in Open Domain Question Answering has evolved since the series of competitions at the Text Retrieval Conference (TREC) [32] and researchers have started adapting neural-network-based QA models for the same tasks. Work done by Yi Tay, Luu Anh, Tuan Siu, Cheung Hui, Jian $\mathrm{Su}$ [33] on Searchqa [29] is ranked highest based on the results achieved on the performance metrics - F1 score and Exact Match (EM) for Open-Domain Question Answering on Searchqa. Work done by Shuohang Wang, Mo Yu, Jing Jiang, et al. [34] is ranked highest for Open-Domain Question Answering on Quasar. Work by Danqi Chen, Adam Fisch, Jason Weston, Antoine Bordes [35] is significant where their approach incorporates a search component based on the combination of bigram hashing and TF-IDF matching with a multilayer recurrent neural network model to detect answers for questions in Wikipedia Paragraphs. 
Table 1. QAS features and techniques. adapted from [9]

\begin{tabular}{|c|c|c|}
\hline QA Features & Paper & Techniques \\
\hline \multirow{4}{*}{$\begin{array}{l}\text { NLIDB:(Natura } \\
\text { Language } \\
\text { Interfaces to } \\
\text { Databases) }\end{array}$} & PRECISE [10] & Identifying classes of \\
\hline & \begin{tabular}{|c|} 
Formal \\
semantic \\
approach [11]
\end{tabular} & $\begin{array}{c}\text { Intermediate representation } \\
\text { language }\end{array}$ \\
\hline & $\begin{array}{c}\text { MASQUE/SQL } \\
{[12]}\end{array}$ & $\begin{array}{l}\text { Portable NL front end to } \\
\text { SQL databases }\end{array}$ \\
\hline & $\begin{array}{c}\text { BASEBALL } \\
{[13]}\end{array}$ & Specific domain Systems \\
\hline \multirow{2}{*}{$\begin{array}{l}\text { Open Domain } \\
\text { Question } \\
\text { Answering }\end{array}$} & $\begin{array}{c}\text { LASSO [14]- } \\
{[17]}\end{array}$ & $\begin{array}{c}\text { Deep linguistic analysis and } \\
\text { iterative strategy }\end{array}$ \\
\hline & FALCON [18] & $\begin{array}{l}\text { Hierarchies of question types } \\
\text { based on the types of } \\
\text { answers sought }\end{array}$ \\
\hline \multirow[t]{2}{*}{ Over text } & DIMAP [19] & $\begin{array}{c}\text { Semantic categories of } \\
\text { answers are mapped into } \\
\text { categories covered by a NE } \\
\text { Recognizer. }\end{array}$ \\
\hline & Mulder [20] & $\begin{array}{l}\text { Extracting "semantic relation } \\
\text { triples" after the document is } \\
\text { parsed, } \\
\text { converting the document into } \\
\text { triples. }\end{array}$ \\
\hline \multirow[t]{4}{*}{ Ontology based } & AquaLog [21] & $\begin{array}{l}\text { Allows the user to choose an } \\
\text { ontology and then ask NL } \\
\text { queries with respect to } \\
\text { the universe of discourse } \\
\text { covered by the ontology }\end{array}$ \\
\hline & $\begin{array}{l}\text { PowerAqua } \\
\text { [22] }\end{array}$ & $\begin{array}{l}\text { QAS focusing on querying } \\
\text { multiple semantic Web } \\
\text { resources }\end{array}$ \\
\hline & \begin{tabular}{|l} 
DeepQA IBM \\
Watson's \\
system [23]
\end{tabular} & $\begin{array}{c}\text { Using unstructured and } \\
\text { structured data (RDF format) } \\
\text { to extract and score evidence }\end{array}$ \\
\hline & PANTO [24] & $\begin{array}{c}\text { Translates factual wh-queries } \\
\text { into F-logic or SPARQL } \\
\text { andevaluates them with } \\
\text { respect to a given KB }\end{array}$ \\
\hline
\end{tabular}

2) Closed-Domain Question Answering: Closed domain Question Answering system involves restricted information sources and domain specific questions. Thus, limited number of questions can be asked. The quality, precision and exactness of answers in closed domain is high. Closed domain data sources include unstructured data, structured data and semi structured data like XML, JSON-annotated texts. Use of domain specific taxonomy is done in closed domain. This domain includes a linguistic requirement to understand the text of the natural language in order to provide correct responses to queries. These domains include temporal QA systems, geospatial QA systems, medical QA systems, patent QA systems and community QA systems. Closed domain QA systems can be combined to create an Open domain QA system [36] [37]. The difference between open and closed domain QA systems is the presence of domain-dependent information that can be used to better the accuracy of the system [38]. Work by [39] proposed methodology of applying semantic information to improve the precision of the information retrieval module in a closed-domain questionanswering system. Felix Mikaelian,' Andre Farias et al. built an end-to-end closed domain question 'answering system built on top of the HuggingFace transformers library [40].

\subsection{Based on Question Type}

In QA systems, the taxonomy of questions posed directly affects the responses. Classification of QA systems based on question types queried by users was first formulated by Mishra et al. [41]. This work classifies based on all the possible types of questions it identifies from literature. The classes types are: list questions, factoid questions, causal questions, confirmation questions and hypothetical type questions.

1) Factoid type questions: These questions are factual in nature and they refer to a single answer [36]. For instance, Who is known as the Father of Nation? Factoid question are wh-type questions belonging to one of these - [what, when, which, who, how].

2) List type questions: The response to a list query is a list of entities or facts in answers. For instance, list name of products available at a price less than $5 \mathrm{k}$ ? List type questions are considered as a series of factoid questions.

3) Hypothetical type questions: Questions having information associated to any assumed event are called hypothetical questions. For instance, questions like 'what would happen if' fall under the category of Hypothetical questions.

4) Causal type questions: Causal questions require explanations on entities they contain. Factoid questions extract answers as named entity, whereas causal questions don't. Pragmatic or discourse level analysis is required for the answer extraction using natural language processing. [42]- [46]

5) Confirmation type questions: Answers to the confirmation type questions are in the form of boolean response - Yes or No, True or False.

\section{MOST FREQUENTLY APPLIED QUESTION ANSWERING TECHNIQUES}

Researchers have used methods, algorithms, frameworks and systems related to knowledge extraction, natural language processing and machine learning to incorporate the modules of a QA architecture.

\subsection{Deep Neural Networks}

Recent developments in deep neural network models have shown promising results in Question Answering. With a small pipeline, these systems require a lot of training. Recurrent Neural Networks such as Gated Recurrent Unit (GRU) and Long Short-term memory (LSTM) help handling long textual matter. With use of attention mechanisms and memory networks the performance of the system can be further enhanced resulting into state-of-the-art performance for deeplearning based Question Answering. Work by [48] incorporates use of a convolutional neural network architecture for reranking pairs of short texts. Work by [49], 
[50] incorporates use of natural language strings to automatically assemble neural networks from a collection of composable modules. Li Dong et al introduces multi-column convolutional neural networks (MCCNNs) to understand questions from three different aspects (namely, answer path, answer context, and answer type) and learn their distributed representations [51]. To recover from local maxima corresponding to incorrect answers Caiming Xiong, Victor Zhong, Richard Socher introduced Dynamic Coattention Network (DCN) for question answering [52], where an iterative procedure involving fusion of co-dependent representations of the question and the document is used to focus on relevant parts of both [53].

Table 2: Leaderboard as of December 2019

\begin{tabular}{|c|c|c|}
\hline DATASET & BEST METHOD & PAPER TITLE \\
\hline SearchQA & DecaProp & $\begin{array}{c}\text { Densely Connected } \\
\text { Attention Propagation for } \\
\text { Reading Comprehension }\end{array}$ \\
\hline Quasar & $\begin{array}{c}\text { Evidence } \\
\text { Aggregation via } \\
\text { R`3 Re-Ranking }\end{array}$ & $\begin{array}{c}\text { Evidence Aggregation for } \\
\text { Answer Re-Ranking in } \\
\text { Open-Domain Question } \\
\text { Answering }\end{array}$ \\
\hline SQuAD1.1 & DrQA & $\begin{array}{c}\text { Reading Wikipedia to } \\
\text { Answer Open-Domain } \\
\text { Questions }\end{array}$ \\
\hline
\end{tabular}

\subsection{Graph Based}

Our thanks to the experts who have contributed towards Gao have described a query graph that is similar to the knowledge base subgraphs and can be mapped directly to a logical form [54]. In their work, semantic parsing is reduced to the generation of query graphs, formulated as a problem of staged search and their method leverages knowledge base for pruning the search space at an early stage and thus simplifies the issue of semantic matching. Research by [55] proposes to enhance the visual answering of questions (VQA) with organized representations of both scene material and queries, using graphs over scene objects and query terms, and a deep neural network that exploits the structure in these representations. To address noisy expressions in questions and problem of questions involving multi-hop logic reasoning on the information graph to get answers, Yuyu Zhang et al. proposed an end-to-end variational learning algorithm which can handle noise in questions, and learn multi-hop reasoning simultaneously [56]. Junwei Bao et al. released a new data-set Complex Questions, aiming to measure the quality of KBQA systems on 'multi-constraint' questions which require multiple knowledge base relations to get the answer and proposed systematic KBQA method using multi-constraint query graph to answer multi-constraint questions [57].
Table 3: Open-Domain Question Answering On Quasar

\begin{tabular}{|c|c|c|c|c|c|}
\hline Rank & $\mathrm{F} 1$ & EM & Method & Paper Title & Year \\
\hline 1 & 42.3 & 49.6 & \begin{tabular}{|c|} 
Evidence \\
Aggregation via \\
R`3 Re-Ranking
\end{tabular} & $\begin{array}{l}\text { Evidence Aggregation } \\
\text { for Answer Re-Ranking } \\
\text { in Open-Domain } \\
\text { Question Answering }\end{array}$ & 2017 \\
\hline 2 & 42.2 & 49.3 & 3 Denoising QA & $\begin{array}{c}\text { Denoising Distantly } \\
\text { Supervised Open- } \\
\text { Domain Question } \\
\text { Answering }\end{array}$ & 2018 \\
\hline 3 & 38.6 & 46.9 & DecaProp & $\begin{array}{l}\text { Densely Connected } \\
\text { Attention Propagation } \\
\text { for Reading } \\
\text { Comprehension }\end{array}$ & 2018 \\
\hline 5 & 26.4 & 26.4 & GA & $\begin{array}{l}\text { Gated-Attention Readers } \\
\text { for Text Comprehension }\end{array}$ & 2016 \\
\hline 6 & 25.9 & 28.5 & BiDAF & $\begin{array}{l}\text { Bidirectional Attention } \\
\text { Flow for Machine } \\
\text { Comprehension }\end{array}$ & 2016 \\
\hline
\end{tabular}

\subsection{Named Entity Recognition}

Recent textual question answering systems have named entity recognition as the important element for information extraction. Diego Molla', Menno van Zaanen and Daniel Smith proposed a named entity recognition system that allows multiple labels to entities giving high recall. [58] Traditional QA systems tend to cut down the size of data. Filtration of documents is done until right answer to the question is found which is achieved by removing the irrelevant piece of information in the documents. So, NER is used to aid this filtration process by getting rid of the information irrelevant to the answer. After analyzing the question and it's type, the answer is mapped to a list of entity types. The textual information is discarded if the entity type is irrelevant to the type of expected answer. [58]. Antonio Toral, Elisa Noguera, Fernando Llopis, and Rafael $\mathrm{Mu}$ noz employed similar approach for Question Anwering task in Spanish texts. [59]

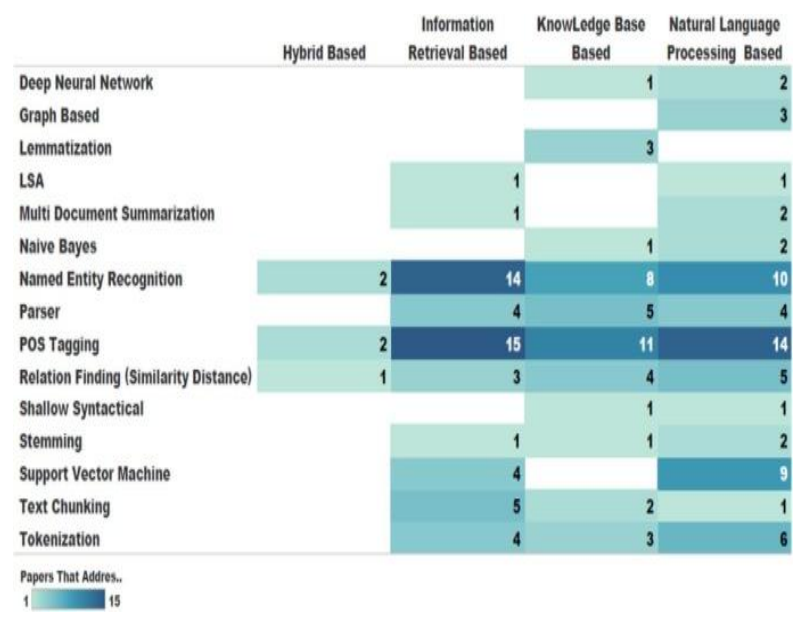

Fig. 2. Frequently used QA Techniques. Adapted from 


\subsection{POS Tagging}

W Wang, J Auer, R Parasuraman et al. hypothesized an approach [60] involving combination of syntactic and semantic features coupled with machine learning techniques for improving accuracy of question answering system on test set of Remedia Corpus [61]. In this approach, the sentences and the factoid questions are preprocessed and tagged by the parts-of-speech tagger distributed with the deep read system [61]. This tagged text is passed to the name identification module and later proper noun identified tagged text is passed to pronoun resolution module. The text is first parsed by the parser before passing it to pronoun resolution module. After this, the sentence question comparison and voting is done. Text with highest scores based on the number of votes is selected as the answer [60]. Another work by Christina Unger, Lorenz Buhmann, Jens Lehmann, Axel-Cyrille Ngonga Ngomo et al. " [62], presents an approach involving parsing of the question and producing a SPARQL template [63] which is identical to the internal structure of the question. This template is then implemented using statistical recognition of entities and predicate detection [62].

\section{EVALUATION METHODS}

With advancements in models and techniques for Question Answering Systems increasing at a rapid rate, evaluation metrics are required for comparing the performance of these methods, models and techniques. According to Yao [64], F1 score and Accuracy are used as the performance metrics in Question Answering. F1 score is calculated on the basis of Precision and Recall. Precision and Recall are calculated using true positive (TP), true negative (TN), false positive (FP) and false negative (FN). These are the fragments of the two classes for which the data is to be classified. Accuracy is the measure of all the correctly identified cases. F1 score is the harmonic mean of Precision and Recall. It is used to give the measure of the incorrectly classified cases.

\section{ACKNOWLEDGEMENTS}

We would like to thank Department of Computer Engineering, Pimpri Chinchwad College of Engineering and Research, Ravet, Pune for their valuable assistance in this literature survey. We would also like to extend our special thanks to Prof. Dr. Archana Chaugule, Head, Department of Computer Engineering, Pimpri Chinchwad College of Engineering and Research, for her encouragement and useful critiques for this research work.

\section{REFERENCES}

[1] E. Cabrio, J. Cojan, F. Gandon, and A. Hallili, "Querying multilingual dbpedia with qakis," in Extended Semantic Web Conference. Springer, 2013, pp. 194-198.

[2] C. Comparot, O. Haemmerle, and N. Hernandez, "An easy way of "expressing conceptual graph queries from keywords and query patterns," in International Conference on Conceptual Structures. Springer, 2010, pp. 84-96.

[3] G. Tsatsaronis, G. Balikas, P. Malakasiotis, I. Partalas, M. Zschunke, M. R. Alvers, D. Weissenborn, A. Krithara, S. Petridis, D. Polychronopoulos et al., "An overview of the bioasq large-scale biomedical semantic indexing and question answering competition," BMC bioinformatics, vol. 16, no. 1, p. 138, 2015.

[4] C. Unger, C. Forascu, V. Lopez, A.-C. N. Ngomo, E. Cabrio, P. Cimiano, and S. Walter, "Question answering over linked data (qald-4)," 2014.
[5] C. Unger, A.-C. N. Ngomo, and E. Cabrio, "6th open challenge on question answering over linked data (qald6)," in Semantic Web Evaluation Challenge. Springer, 2016, pp. 171-177.

[6] R. Usbeck, M. Roder, P. Haase, A. Kozlov, M. Saleem, and A.- " C. N. Ngomo, "Requirements to modern semantic search engine," in International Conference on Knowledge Engineering and the Semantic Web. Springer, 2016, pp. 328-343.

[7] J. Ko, L. Si, and E. Nyberg, "Combining evidence with a probabilistic framework for answer ranking and answer merging in question answering," Information processing \& management, vol. 46, no. 5, pp. 541-554, 2010.

[8] C. Manning, "Text-based question answering systems." p. p. 7., 2013

[9] A. Bouziane, D. Bouchiha, N. Doumi, and M. Malki, "Question answering systems: survey and trends," Procedia Computer Science, vol. 73, pp. 366-375, 2015.

[10] A.-M. Popescu, O. Etzioni, and H. Kautz, "Towards a theory of natural language interfaces to databases," in Proceedings of the 8th international conference on Intelligent user interfaces. ACM, 2003, pp. 149-157.

[11] A. De Roeck, A natural language system based on formal semantics. Universiti Sains Malaysia, 1991.

[12] I. Androutsopoulos, G. D. Ritchie, and P. Thanisch, "Natural language interfaces to databases-an introduction," Natural language engineering, vol. 1, no. 1, pp. 29-81, 1995

[13] W. A. Woods, "Progress in natural language understanding: an application to lunar geology," in Proceedings of the June 4-8, 1973, national computer conference and exposition. ACM, 1973, pp. 441-450.

[14] E. Hovy, L. Gerber, U. Hermjakob, M. Junk, and C.-Y. Lin, "Question answering in webclopedia," in TREC, vol. 52, 2000, pp. 53-56.

[15] M. Wu, X. Zheng, M. Duan, T. Liu, T. Strzalkowski, and S. Albany, "Question answering by pattern matching, web-proofing, semantic form proofing," in NIST Special Publication: The Twelfth Text REtrieval Conference (TREC), 2003, pp. 500-255.

[16] D. Moldovan, S. Harabagiu, M. Pasca, A. Harabagiu, R. Mihalcea, R. Girju, R. Goodrum, and V. Rus, "Lasso: A tool for surfing the answer net," 1999.

[17] R. Srihari and W. Li, "Information extraction supported question answering," CYMFONY NET INC WILLIAMSVILLE NY, Tech. Rep., 1999.

[18] S. Harabagiu, D. Moldovan, M. Pasca, R. Mihalcea, M. Surdeanu, R. Bunescu, R. Girju, V. Rus, and P. Morarescu, "Falcon: Boosting knowledge for answer engines," in TREC, vol. 9, 2000, pp. 479-488.

[19] K. C. Litkowski, "Syntactic clues and lexical resources in questionanswering," NIST SPECIAL PUBLICATION SP, vol. 249, pp. 157-166, 2001.

[20] C. Kwok, O. Etzioni, O. Etzioni, and D. S. Weld, "Scaling question answering to the web," ACM Transactions on Information Systems (TOIS), vol. 19, no. 3, pp. 242-262, 2001.

[21] V. Lopez, V. Uren, E. Motta, and M. Pasin, “Aqualog: 
An ontologydriven question answering system for organizational semantic intranets," Web semantics: science, services and agents on the world wide web, vol. 5, no. 2, pp. 72-105, 2007.

[22] V. Lopez, M. Fernandez, E. Motta, and N. Stieler, "Poweraqua: Sup-' porting users in querying and exploring the semantic web," Semantic Web, vol. 3, no. 3, pp. 249-265, 2012.

[23] A. Kalyanpur, B. K. Boguraev, S. Patwardhan, J. W. Murdock, A. Lally, C. Welty, J. M. Prager, B. Coppola, A. Fokoue-Nkoutche, L. Zhang et al., "Structured data and inference in deepqa," IBM Journal of Research and Development, vol. 56, no. 3.4, pp. 10-1, 2012.

[24] C. Wang, M. Xiong, Q. Zhou, and Y. Yu, "Panto: A portable natural language interface to ontologies," in European Semantic Web Conference. Springer, 2007, pp. 473-487.

[25] A. Allam and M. Haggag, "The question answering systems: A survey," International Journal of Research and Reviews in Information Sciences, vol. 2, pp. 211221, 092012.

[26] B. Dhingra, K. Mazaitis, and W. W. Cohen, "Quasar: Datasets for question answering by search and reading," arXiv preprint arXiv:1707.03904, 2017.

[27] T. Nguyen, M. Rosenberg, X. Song, J. Gao, S. Tiwary, R. Majumder, and L. Deng, "Ms marco: A humangenerated machine reading comprehension dataset," 2016.

[28] M. Joshi, E. Choi, D. S. Weld, and L. Zettlemoyer, "Triviaqa: A large scale distantly supervised challenge dataset for reading comprehension," arXiv preprint arXiv:1705.03551, 2017.

[29] M. Dunn, L. Sagun, M. Higgins, V. U. Guney, V. Cirik, and K. Cho, "Searchqa: A new q\&a dataset augmented with context from a search engine," arXiv preprint arXiv:1704.05179, 2017.

[30] P. Rajpurkar, J. Zhang, K. Lopyrev, and P. Liang, "Squad: 100,000+ questions for machine comprehension of text," arXiv preprint arXiv:1606.05250, 2016.

[31] Z. Yang, P. Qi, S. Zhang, Y. Bengio, W. W. Cohen, R. Salakhutdinov, and C. D. Manning, "Hotpotqa: A dataset for diverse, explainable multi-hop question answering," arXiv preprint arXiv:1809.09600, 2018.

[32] E. M. Voorhees, "Question answering in trec," in Proceedings of the tenth international conference on Information and knowledge management. ACM, 2001, pp. 535-537.

[33] Y. Tay, L. A. Tuan, S. C. Hui, and J. Su, "Densely connected attention propagation for reading comprehension," 2018.

[34] S. Wang, M. Yu, J. Jiang, W. Zhang, X. Guo, S. Chang, Z. Wang, T. Klinger, G. Tesauro, and M. Campbell, "Evidence aggregation for answer re-ranking in opendomain question answering," 2017.

[35] D. Chen, A. Fisch, J. Weston, and A. Bordes, "Reading wikipedia to answer open-domain questions," 2017.

[36] N. Indurkhya and F. J. Damerau, Handbook of natural language processing. Chapman and Hall/CRC, 2010.
[37] V. Lopez, V. Uren, M. Sabou, and E. Motta, "Is question answering fit for the semantic web?: a survey," Semantic Web, vol. 2, no. 2, pp. 125-155, 2011.

[38] D. Molla and J. L. Vicedo, "Question answering in restricted domains: 'An overview," Computational Linguistics, vol. 33, no. 1, pp. 41-61, 2007.

[39] H. Doan-Nguyen and L. Kosseim, "Improving the precision of a closed-domain question-answering system with semantic information," in Coupling Approaches, Coupling Media and Coupling Languages for Information Retrieval, ser. RIAO '04. Paris, France, France: LE CENTRE DE HAUTES ETUDES INTERNATIONALES D'INFORMATIQUE DOCUMENTAIRE, 2004, pp. 850-859. [Online]. Available: http://dl.acm.org/citation.cfm?id=2816272.2816351

[40] M. A. T. N. O. S. Felix Mikaelian, Andr ' e Farias, "cdqa: Closed domain ' question answering - an end-toend closed domain question answering system," https://github.com/cdqa-suite/cdQA, 2019.

[41] A. Mishra and S. K. Jain, "A survey on question answering systems with classification," Journal of King Saud University-Computer and Information Sciences, vol. 28, no. 3, pp. 345-361, 2016.

[42] R. Higashinaka and H. Isozaki, "Corpus-based question answering for why-questions," in Proceedings of the Third International Joint Conference on Natural Language Processing: Volume-I, 2008.

[43] S. Verberne, L. Boves, N. Oostdijk, and P. Coppen, "Discourse-based answering of why-questions," 2007

[44] S. Verberne, L. Boves, N. Oostdijk, and P.-A. Coppen, "Using syntactic information for improving whyquestion answering," in Proceedings of the 22nd International Conference on Computational LinguisticsVolume 1. Association for Computational Linguistics, 2008, pp. 953-960.

[45] _ "What is not in the bag of words for why-qa?" Computational Linguistics, vol. 36, no. 2, pp. 229-245, 2010.

[46] D. Moldovan, S. Harabagiu, M. Pasca, R. Mihalcea, R. Girju, R. Goodrum, and V. Rus, "The structure and performance of an opendomain question answering system," in Proceedings of the 38th annual meeting on association for computational linguistics. Association for Computational Linguistics, 2000, pp. 563-570.

[47] M. A. C. Soares and F. S. Parreiras, "A literature review on question answering techniques, paradigms and systems," Journal of King Saud University-Computer and Information Sciences, 2018.

[48] A. Severyn and A. Moschitti, "Learning to rank short text pairs with convolutional deep neural networks," in Proceedings of the 38th international ACM SIGIR conference on research and development in information retrieval. ACM, 2015, pp. 373-382.

[49] J. Andreas, M. Rohrbach, T. Darrell, and D. Klein, "Learning to compose neural networks for question answering," arXiv preprint arXiv:1601.01705, 2016.

[50] R. M. D. T. K. D. Andreas, Jacob, "Neural module networks," in Proceedings of the IEEE Conference on 
Computer Vision and Pattern Recognition, 2016, pp. 3948.

[51] L. Dong, F. Wei, M. Zhou, and K. Xu, "Question answering over freebase with multi-column convolutional neural networks," in Proceedings of the 53rd Annual Meeting of the Association for Computational Linguistics and the 7th International Joint Conference on Natural Language Processing (Volume 1: Long Papers), 2015, pp. 260-269.

[52] C. Xiong, S. Merity, and R. Socher, "Dynamic memory networks for visual and textual question answering," in International conference on machine learning, 2016, pp. 2397-2406.

[53] C. Xiong, V. Zhong, and R. Socher, "Dynamic coattention networks for question answering," arXiv preprint arXiv:1611.01604, 2016.

[54] S. W.-t. Yih, M.-W. Chang, X. He, and J. Gao, "Semantic parsing via staged query graph generation: Question answering with knowledge base," 2015.

[55] D. Teney, L. Liu, and A. van den Hengel, "Graphstructured representations for visual question answering," in Proceedings of the IEEE Conference on Computer Vision and Pattern Recognition, 2017, pp. 1-9.

[56] Y. Zhang, H. Dai, Z. Kozareva, A. J. Smola, and L. Song, "Variational reasoning for question answering with knowledge graph," in ThirtySecond AAAI Conference on Artificial Intelligence, 2018.

[57] J. Bao, N. Duan, Z. Yan, M. Zhou, and T. Zhao, "Constraint-based question answering with knowledge graph," in Proceedings of COLING 2016, the 26th International Conference on Computational Linguistics: Technical Papers, 2016, pp. 2503-2514.

[58] D. Molla, M. van Zaanen, and D. Smith, "Named entity recognition ' for question answering," in Proceedings of the Australasian Language Technology Workshop 2006, Sydney, Australia, Nov. 2006, pp. 51-58. [Online]. Available: https://www.aclweb.org/anthology/U06-1009

[59] A. Toral, E. Noguera, F. Llopis, and R. Munoz, "Improving question answering using named entity recognition," in International Conference on Application of Natural Language to Information Systems. Springer, 2005, pp. 181-191

[60] W. Wang, J. Auer, R. Parasuraman, I. Zubarev, D. Brandyberry, and M. Harper, "A question answering system developed as a project in a natural language processing course," in Proceedings of the 2000 ANLP/NAACL Workshop on Reading comprehension tests as evaluation for computer-based language understanding sytems-Volume 6. Association for Computational Linguistics, 2000, pp. 28-35.

[61] L. Hirschman, M. Light, E. Breck, and J. D. Burger, "Deep read: A reading comprehension system," in Proceedings of the 37th annual meeting of the Association for Computational Linguistics on Computational Linguistics. Association for Computational Linguistics, 1999, pp. 325-332.

[62] C. Unger, L. Buhmann, J. Lehmann, A.-C. Ngonga Ngomo, D. Gerber, " and P. Cimiano, "Template-based question answering over rdf data," in Proceedings of the 21st International Conference on World Wide Web, ser. WWW '12. New York, NY, USA: ACM, 2012, pp. 639648. [Online]. Available: http://doi.acm.org/10.1145/2187836.2187923

[63] O. Corby, C. F. Zucker, and F. Gandon, "Sparql template: a transformation language for rdf," 2014.

[64] X. Yao, "Feature-driven question answering with natural language alignment," Ph.D. dissertation, Johns Hopkins University, 2014.," unpublished. 